\title{
NEFROTOXICIDADE INDUZIDA POR RADIOCONTRASTE EM RATOS WISTAR FÊMEAS UNINEFRECTOMIZADOS E EM RESTRIÇÃO HÍDRICA
}

\author{
Valéria Cristina da Rocha Campos ${ }^{1}$, Janaína Luise Mendes Vimeney ${ }^{2}$, Felipe Lopes \\ Campos $^{1}$, Ana Maria Reis Ferreira ${ }^{3}$, Maria Lúcia Ribeiro Caldas ${ }^{3}$, \\ Heloísa Werneck de Macedo ${ }^{3}$

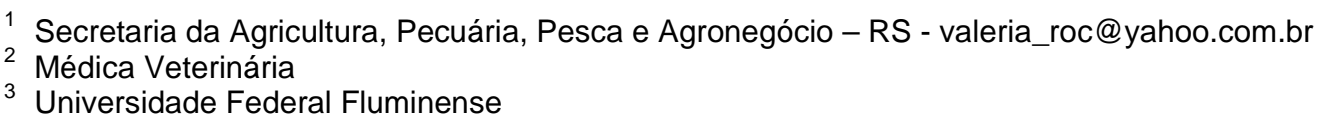

RESUMO: O presente estudo avaliou os danos renais causados pela administração dos contrastes radiológicos diatrizoato de sódio, iônico e hiperosmolar e do lopamidol, não iônico e de baixa osmolaridade, em ratos Wistar fêmeas uni-nefrectomizados e em restrição hídrica. Foram utilizados 22 ratos Wistar fêmeas, sendo divididos em quatro grupos de acordo com o tratamento efetuado: $\mathrm{Gl}$ - salina $(n=5)$, GII - Sham ( $n=5)$, GIII - diatrizoato de sódio $(n=5)$ e GIV - lopamidol $(n=7)$. Os efeitos produzidos pelos contrastes radiológicos foram avaliados pela dosagem de creatinina sérica e pela histopatologia renal descritiva e semiquantitativa. No 18ำ dia do experimento, três horas após aplicação intravenosa do contraste diatrizoato (GIII) houve um aumento significativo nos níveis de creatinina sérica em relação aos grupos controles salina (GI) e Sham (GII). Após a administração do lopamidol (GIV) houve um decréscimo no nível de cretinina sérica. No entanto, comparando-se os grupos diatrizoato (GIII) e lopamidol (GIV), no 18을. não foi notada diferença estatística na função renal. A vacuolização das células dos túbulos renais foi acentuada (3+) tanto no grupo que recebeu o contraste diatrizoato quanto no grupo lopamidol. Com base na função renal, neste experimento, o contraste de baixa osmolaridade e não iônico mostrou-se tão nefrotóxico quanto o de alta osmolaridade e iônico.

Palavras-chave: creatinina sérica; diatrizoato; fêmea; histopatologia; lopamidol; rato

\section{CONTRAST MEDIA-INDUCED NEPHROTOXICITY IN UNINEPHRECTOMIZED AND DEHYDRATED FEMALE WISTAR RATS}

ABSTRACT: The present study evaluated renal injury induced by contrast media diatrizoate and lopamidol in uninephrectomized and dehydrated female Wistar rats. Twenty two female Wistar rats were divided into four groups according to the treatment performed: $\mathrm{Gl}$ - Saline $(n=5)$, Gll - Sham $(n=5)$, GIII - diatrizoate $(n=5)$ and GIV - lopamidol $(n=7)$. The effects induced by contrast media were evaluated by seric levels of creatinine and by descriptive and semi-quantitative renal histopathology. On the eighteenth day of the experiment, three hours after intravenuous application of contrast media diatrizoate, group GllI has presented increased level of serum creatinine compared to saline (GI) and Sham (GII) control groups. After administration of lopamidol, group GIV has presented decreased serum creatinine levels. However when comparing diatrizoate group (GIII) and lopamidol group (GIV), on the eighteenth day, there was no statistical difference in renal function between them. Proximal tubular cells vacuolization was proeminent $(3+)$ in lopamidol and diatrizoate group. According to renal function evaluation, low-osmolar contrast media was as nephrotoxic as the high-osmolar one.

Key words: diatrizoate; female; histophatology; lopamidol; rat; serum creatinine 


\section{INTRODUÇÃO}

Uma das complicações do uso de radiocontrastes em humanos e animais é a ocorrência de nefropatia induzida por contraste (NCR). Esta é definida por declínio na função renal em seguida à administração intravenosa de contraste radiológico (Morcos, 2005), pois os agentes de contraste radiológicos intravenosos são conhecidos como substâncias nefrotóxicas (Nelson e Couto, 2006). Normalmente, um indivíduo clinicamente saudável consegue eliminar essas substâncias principalmente por via renal. No entanto, frente a uma doença renal pré-existente ou à existência de fatores de risco, esses compostos podem se acumular no organismo ocasionando insuficiência renal aguda. Vários fatores de risco, como exemplo o tipo de radiocontraste administrado e o sexo do indivíduo, influenciam o desenvolvimento da NCR (lakovou et al., 2003; Aspelin et al., 2003).

Em pacientes com redução da função ou da perfusão renal, as partículas que constituem os meios de contrastes radiológicos induzem mudanças funcionais e estruturais em sua passagem pelos rins. $O$ dano renal é evidenciado por vacuolização citoplasmática e perda da borda em escova das células dos túbulos contorcidos proximais (TP), causadas por toxicidade tubular direta (Morcos, 2005). Estudos experimentais mostraram que a injeção intravascular de meio de contraste em ratos causa vacuolização citoplasmática em células dos túbulos renais proximais, além de produzir um aumento nos níveis de uréia e creatinina séricas (Çaglar et al., 2001). Em ratos, a formação da vacuolização tubular renal proximal se desenvolve completamente em 2 a 3,5 horas depois da administração do contraste (Tervahartiala et al., 1991).
O estresse oxidativo, a hiper-
osmolaridade e os efeitos
hemodinâmicos são citados como
mecanismo da toxicidade dos
radiocontrastes (Haron et al., 2009). O
mecanismo celular da citotoxicidade dos
agentes de contraste pode estar
relacionado em parte às suas propriedades físico-químicas, particularmente sua hiperosmolaridade e carga iônica (Schick e Haller, 1999). A administração intravenosa dos meios de contraste iodados de alta osmolaridade e iônicos, como o diatrizoato de sódio, pode ocasionar em alguns casos, efeitos adversos como aumento da viscosidade sanguínea, dano endotelial, hipervolemia, vasodilatação, edema, depressão miocardial, vômito e convulsão (Viana et al., 2000). Sendo assim, os radiocontrastes não iônicos e de baixa osmolaridade foram desenvolvidos com o objetivo de reduzir a incidência desses efeitos indesejáveis (Soejima et al., 2003). O lopamidol, radiocontraste não iônico e de baixa osmolaridade, é muito utilizado em medicina veterinária, sendo empregado principalmente para mielografia. Vários estudos citam o lopamidol como sendo menos nefrotóxico que o diatrizoato (Cavaliere et al., 1987; Katholi et al., 1993).

Quanto ao sexo, a literatura já cita o sexo feminino como mais predisposto à supracitada nefropatia (Gill et al., 2004; Habeb et al., 2005). Como essa nefrotoxicidade ocorre quando há uma doença renal pré-existente, em estudos experimentais, se faz necessária a utilização de métodos como a nefrectomia e a desidratação para tornar os animais mais suscetíveis à ação nefrotóxica destes fármacos (Wang et al., 2001).

Diante da escassez de trabalhos experimentais realizados com ratos fêmeas e de controvérsias entre 
achados clínicos e experimentais quanto à nefrotoxicidade induzida pelos agentes de contraste iodado de alta e de baixa osmolaridade, 0 presente estudo objetivou avaliar comparativamente os danos renais causados pelo uso do diatrizoato de sódio - iônico e de alta osmolaridade - e do lopamidol - não iônico e de baixa osmolaridade em ratos fêmeas Wistar uninefrectomizados e em restrição hídrica.

\section{MATERIAL E MÉTODOS}

Foram utilizados 22 ratos Wistar fêmeas (148,95 g \pm 34,25 g), sendo divididos em quatro grupos de acordo com o tratamento efetuado: GI - salina $(\mathrm{NaCl} 0,9 \% ; 7,8 \mathrm{~mL} / \mathrm{Kg} ; \mathrm{n}=5)$; Gll Sham ( $n=5)$; GIII - diatrizoato de sódio (Urografina $^{\circledR} 370 ; 7,8 \mathrm{~mL} / \mathrm{Kg}(2,9 \mathrm{~g} \mathrm{I} / \mathrm{Kg})$; $\mathrm{n}=5$ ) e GIV - lopamidol (lopamiron ${ }^{\circledR}$ $370 ; 7,8 \mathrm{~mL} / \mathrm{Kg}(2,9 \mathrm{~g} \mathrm{l} / \mathrm{Kg}) ; \mathrm{n}=7)$.

Os animais foram mantidos no Biotério de Patologia Experimental do Departamento de Patologia - UFF, em ciclo de $12 \mathrm{~h}$ claro/escuro e temperatura entre 20 a $23^{\circ} \mathrm{C}$. Receberam ração ad libitum e água, exceto no período de restrição hídrica. A manipulação experimental foi iniciada após período de adaptação de pelo menos uma semana.

No primeiro dia do experimento, os animais foram pesados, anestesiados com quetamina (Ketamin-S $(+)^{\circledR} 50$ $\mathrm{mg} / \mathrm{mL}, \quad 80 \mathrm{mg} / \mathrm{kg}$, IM) e xilazina (Virbaxyl $^{\circledR} 2 \%, 8 \mathrm{mg} / \mathrm{kg}$, IM), submetidos à nefrectomia unilateral esquerda (UNX) e identificados. Após a retirada do rim esquerdo, o mesmo foi seccionado longitudinalmente, fixado em Dubosq Brazil por $4 \mathrm{~h}$, pós-fixado em formalina a $10 \%$ por $24 \mathrm{~h}$ e processado de acordo com a rotina histológica para inclusão em parafina. O rim esquerdo foi avaliado como controle. Enquanto que no grupo Sham, realizou-se apenas uma simulação da nefrectomia, ou seja, foi realizada a lombotomia subcostal de aproximadamente $3 \mathrm{~cm}$ de extensão e o rim esquerdo foi apenas manipulado, não sendo removido.

No $16^{\circ}$ dia, os animais foram pesados, anestesiados e procedeu-se coleta de $1 \mathrm{~mL}$ de sangue através de punção cardíaca, para determinação dos níveis séricos basais de creatinina por sistema colorimétrico com reação de ponto final.

No $17^{\circ}$ dia, foi feita restrição hídrica dos animais por $24 \mathrm{~h}$. Após esse período, a água foi novamente disponibilizada, os animais foram pesados e anestesiados, para facilitar a contenção dos mesmos e principalmente para a administração intravenosa dos contrastes radiológicos, que deve ser feita lentamente. Procedeu-se administração dos fármacos de acordo com os grupos experimentais, por meio de scalp BD 27, na veia caudal durante 3 minutos. Três horas depois, os animais foram anestesiados e nova coleta de $1 \mathrm{~mL}$ de sangue foi feita para determinação dos níveis de creatinina. $\mathrm{Em}$ seguida, as ratas foram eutanasiadas através de injeção intracardíaca de quetamina $(80 \mathrm{mg} / \mathrm{Kg})$ e necropsiadas mediante abertura das cavidades toraco-abdominal. Fragmentos de tecido renal foram fixados em Dubosq Brazil por $4 \mathrm{~h}$, pós-fixados em formalina a $10 \%$ por $24 \mathrm{~h}$ e processados de acordo com a rotina histológica para inclusão em parafina. Os cortes foram realizados à espessura de $3 \mu \mathrm{m}$, corados por Hematoxilina e Eosina (HE) e Ácido Periódico de Schiff (PAS) e examinados em microscópio Nikon Eclipse $\mathrm{E} 400^{\circledR}$.

$\mathrm{Na}$ análise descritiva, foi avaliada a presença de vacuolização citoplasmática tubular, sendo classificada como leve, moderada e acentuada. A análise semiquantitativa foi feita graduando-se as alterações tubulares em escala arbitrária de $0+$ a $3+$, assim definidas: $0+$ ausência de lesão; 1+ alterações evidentes, raras e focais; 2 + alterações 
proeminentes, acometendo menos de $50 \%$ do tecido cortical examinado e $3+$ alterações proeminentes, acometendo mais de $50 \%$ do tecido cortical examinado.

Para análise estatística foi utilizado o programa estatístico SPSS vs.10. Os resultados de creatinina foram avaliados através do Teste t de Student e ANOVA, para comparação entre os tratamentos efetuados, sendo adotado nível de significância de $5 \%(P<0,05)$. A moda, que é o valor mais frequente observado, foi empregada para a definição do grau de lesão de cada grupo estudado obtido pela análise semiquantitativa das alterações histopatológicas.

\section{RESULTADOS E DISCUSSÃO}

Nos grupos controles salina (GI) e Sham (GII) não houve diferença significativa nos valores de creatinina sérica entre o $16^{\circ}$ dia e o $18^{\circ}$ dia do experimento (Tabela 1).

No $18^{\circ}$ dia, após três horas da aplicação intravenosa do diatrizoato de sódio (GIII), constatou-se uma elevação significativa no nível de creatinina, em comparação aos grupos controles. Resultado semelhante foi encontrado por Duan et al. (2009). Após a administração do lopamidol (GIV) houve um decréscimo no nível de cretinina sérica, o que confere com resultados de outros estudos, que enfatizam que os contrastes radiológicos de baixa osmolaridade e não iônicos são menos nefrotóxicos (Walday et al., 1995; Schick e Haller, 1999). Porém, comparando-se os dois contrastes radiológicos (GIII e GIV), no $18^{\circ}$ dia, não foi notada diferença significativa nos níveis de creatinina sérica entre esses grupos. Esse resultado também foi encontrado em alguns estudos clínicos humanos (Evans e Cutler, 1987; Moore et al., 1992; Cochran et al., 1988). Nenhuma diferença também foi relatada por Schwab et al. (1989) em um estudo clínico sobre a incidência de leve disfunção renal em pacientes de alto risco expostos ao lopamidol ou ao diatrizoato. O grupo salina (GI) não se diferenciou do grupo lopamidol (GIV). Duan et al. (2009) também verificou não haver diferença significante no nível de creatinina sérica entre o grupo iohexol e - grupo controle, ao avaliar a nefrotoxicidade do diatrizoato (alta osmolaridade) e do iohexol (baixa osmolaridade). O grupo Sham (GII) se destacou dos demais grupos, por apresentar valores baixos de creatinina no $18^{\circ}$ dia (Tabela 1 ).

A lesão de NCR mais frequente tanto em estudos experimentais quanto em casos clínicos é a vacuolização do TP. Neste estudo, os grupos salina (GI), Sham (GII) e diatrizoato de sódio (GIII) receberam grau $0+$ (Tabela 2 ), o que significou ausência de vacuolização na amostra renal proveniente da nefrectomia unilateral esquerda. Enquanto o grupo lopamidol (GIV) apresentou leve vacuolização (grau 1+) na amostra renal esquerda. Este é um achado normal da morfologia renal de ratos, segundo Tervahartiala et al. (1997).

As amostras renais do grupo salina (GI) e do grupo Sham (GII) receberam grau $0+$ no $18^{\circ}$ dia do experimento. Foi constatada presença acentuada de microvacúolos, de diferentes tamanhos, na sua maioria apicais, no epitélio do TP após a aplicação de ambos os contrastes estudados, sendo os grupos diatrizoato de sódio (GIII) e lopamidol (GIV) classificados em grau 3+. Thomsen et al. (1988) constatou não haver mudanças na microscopia óptica de rins entre os contrastes de baixa osmolaridade e de alta osmolaridade em ratos Wistar machos. Já Duan et al. (2009), ao utilizar outro modelo experimental, observou que o contraste radiológico de baixa osmolaridade foi menos tóxico aos rins dos ratos Sprague-Dawley que o contraste de alta 
Tabela 1 - Comparação dos níveis séricos de creatinina dos grupos salina, sham, diatrizoato e lopamidol em ratos

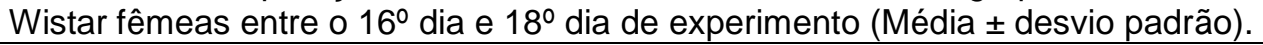

\begin{tabular}{ccccccccc}
\hline \multirow{2}{*}{ Grupo } & \multicolumn{2}{c}{ GI } & \multicolumn{2}{c}{ Gll } & \multicolumn{2}{c}{ Glll } & \multicolumn{2}{c}{ GIV } \\
\cline { 2 - 8 } & $16^{\circ}$ dia & $18^{\circ}$ dia & $16^{\circ}$ dia & $18^{\circ}$ dia & $16^{\circ}$ dia & $18^{\circ}$ dia & $16^{\circ}$ dia & $18^{\circ}$ dia \\
\hline $\begin{array}{c}\text { Cretinina } \\
\text { (mg/dL) }\end{array}$ & $0,66 \pm 0,09^{\mathrm{a}}$ & $0,57 \pm 0,06^{\mathrm{a}}$ & $0,57 \pm 0,22^{\mathrm{a}}$ & $0,37 \pm 0,10^{\mathrm{a}}$ & $0,46 \pm 0,02^{\mathrm{a}}$ & $0,78 \pm 0,02^{\mathrm{b}, \mathrm{a}}$ & $0,81 \pm 0,10^{\mathrm{b}}$ & $0,62 \pm 0,10^{\mathrm{a}}$ \\
\hline
\end{tabular}

Letras em sobrescrito: letras iguais são estatisticamente iguais e para letras diferentes tem-se que "b" é estatisticamente superior a "a". "b, a", onde a primeira letra representa a comparação entre o 16ำ e 18을 dia dentro do mesmo grupo e a segunda letra representa a comparação entre os grupos GIII e GIV, no 18ํำ dia do experimento. GI - salina; GII - Sham; GIII - diatrizoato de sódio e GIV - lopamidol.

Tabela 2 - Análise semiquantitativa da vacuolização do túbulo renal proximal de acordo com os grupos experimentais

\begin{tabular}{ccc}
\hline Grupo & \multicolumn{2}{c}{ Vacuolização citoplasmática tubular } \\
\cline { 2 - 3 } & $\mathrm{RE}(\mathrm{UNX} /$ Sham $)$ & $\mathrm{RD}\left(18^{\circ}\right.$ dia $)$ \\
\hline GI & $0+$ & $0+$ \\
GII & $0+$ & $0+$ \\
GIII & $0+$ & $3+$ \\
GIV & $1+$ & $3+$ \\
\hline
\end{tabular}

GI - salina; GII - Sham; GIII - diatrizoato de sódio e GIV - lopamidol. RE - rim esquerdo; RD - rim direito; UNX - nefrectomia unilateral.

osmolaridade. Embora sejam observadas alterações lisossomais nas células do TP, estas não parecem refletir consequências funcionais renais (Heyman et al., 1988). A vacuolização é considerada um sinal precoce e reversível do dano celular. Ela parece ser um sinal mais sensível, aparecendo logo depois da administração do radiocontraste (Tervahartiala et al., 1997).

No presente estudo, houve correlação entre os achados bioquímicos e os histopatológicos após a aplicação do contraste diatrizoato e do lopamidol. Embora, alguns estudos afirmem que o grau de vacuolização não está correlacionado com a perda de função renal (Walday et al., 1995) e que as alterações vacuolares dependam do tipo de contraste administrado (Tervahartiala, 1997).

\section{CONCLUSÃO}

A administração intravenosa do contraste radiológico diatrizoato de sódio produziu um aumento significativo no nível de creatinina sérica em comparação com os grupos controles salina e Sham. O lopamidol produziu decréscimo no nível de creatinina no $18^{\circ}$ dia. Nos grupos que receberam os contrastes radiológicos, observou-se acentuado grau de vacuolização (3+) nos túbulos renais proximais e não houve diferença significativa nos níveis de creatinina sérica entre esses grupos, no $18^{\circ}$ dia do experimento. O contraste de baixa osmolaridade e não iônico mostrou-se tão nefrotóxico quanto o de alta osmolaridade e iônico.

\section{NOTA INFORMATIVA}

Número do protocolo no Comitê de Ética e Pesquisa CMM/HUAP n ${ }^{\circ} 166 / 05$ em 07/10/2005.

\section{REFERÊNCIAS}

ASPELIN, P.; AUBRY, P.; FRANSSON, S-G. et al. Nephrotoxic effects in High-risk patients undergoing angiography. New England Journal of Medicine, v. 348, p.491-499, 2003.

CAVALIERE, G.; ARRIGO, G.; D'AMICO, G. et al. Tubular nephrotoxicity after intravenous urography with ionic hight-osmolal and nonionic low-osmolal contrast media in patients with 
chronic renal insufficiency. Nephron, v.46, n.2, p.128-133, 1987.

COCHRAN, S.T.; BALLARD, J.W.; KATZBERG, R.W. et al. Evaluation of lopamidol and diatrizoate in excretory urography: a double-blind clinical study. American Journal of Roentgenology, v.151, p.523-527, 1988.

ÇAGLAR, Y.; METE, U.Ö.; KAYA, M. Ultrastrutuctural evaluation of the effects of the contrast media on the rat kidney. Journal of Submicroscopic Cytology and Pathology, v.33, v.4, p.443-451, 2001.

DUAN, S.B.; WANG, Y.H.; LIU, F.Y. et al. The protective role of Telmisartan against nephrotoxicity induced by $X$ - ray contrast media in rat model. Acta Radiologica, v.50, p.754-759, 2009.

EVANS, J.R., CUTLER, R.E. Low-osmolar radiocontrast agents and nephrotoxicity. Nephrology Dialysis Transplantation, v.17, p.504-508, 1987.

GILL, N.K.; PICCIONE, E.A.; VIDO, D.A. et al. Gender as a risk factor for contrast nephropathy: Effects of hydration and N-Acetylcysteine. Clinical Cardiology, v.27, p.554-558, 2004.

HABEB, M.; AGAC, M.T.; ALIYEV, F. et al. Contrast media-induced nephropathy: clinical burden and current attempts for prevention. Anadolu Kardiyoloji Dergisi, v.5, p.124 -129, 2005.

HARMON, R.C.; DUFFY, S.P.; TERNEUS, M.V. et al. Characterization of a novel model for investigation of radiocontrast nephrotoxicity. Nephrology Dialysis Transplantation, v. 24, p. 763-768, 2009.

HEYMAN, S.N. ; BREZIS, M. ; REUBINOFF, C.A. et al. Acute renal failure with selective medullary injury in the rat. The Journal of Clinical Investigation, v.82, p. 401-412, 1988.

IAKOVOU, I.; DANGAS, G; MEHRAN, R. et al. Impact of gender on the incidence and outcome of contrast -induced nephropathy after percutaneous coronary intervention. Journal of Invasive Cardiology, v.15, n.1, p.18-22, 2003.

KATHOLI, R.E.; TAYLOR, G.J.; WOODS, W.T. et al. Nephrotoxicity of nonionic low-osmolality versus ionic high-osmolality contrast media: A prospective double-blind randomized comparision in human beings. Radiology, v.186, p.183-187, 1993.

MOORE, R.D.; STEINBERG, E.P.; POWE, N.R. et al. Nephrotoxicity of high-osmalality versus low-osmolality contrast media: randomized clinical trial. Radiology, v.182, p.649-655, 1992.
MORCOS, S.K. Prevention of contrast mediainduced nephrotoxicity after angiographic procedures. Journal of Vascular and Interventional Radiology, v.16, p.13-23, 2005.

NELSON, R.W.; COUTO, C.G. Medicina Interna de Pequenos Animais. Rio de Janeiro: Elsevier, 2006. 1324p.

SCHICK, C.S.; HALLER, C. Comparative cytotoxicity of ionic and non-ionic radiocontrast agents on MDCK cell monolayers in vitro. Nephrology Dialysis Transplantation, v.14, p.342-347, 1999.

SCHWAB, S.J.; HLATKY, M.A.; PIEPER, K.S. et al. Contrast nephrotoxicity: a randomized controlled trial of a nonionic ando ionic radiographic contrast agent. The New England Journal of Medicine, v.320, p.149-153, 1989.

SOEJIMA, K.; UOZUMI, J.; KANOU, T. et al. Nonionic contrast media are less nephrotoxic than ionic contrast media to rat renal cortical slices. Toxicology Letters, v.143, p.17-25, 2003.

TERVAHARTIALA, P.; KIVISAARI, L.; KIVISAARI, R. et al. Contrast media-induced renal tubular vacuolization. A light and electron microscopic study on rat kidneys. Investigative Radiology, v.26, p.882-887, 1991.

TERVAHARTIALA, P.; KIVISAARI, L.; KIVISAARI, R. et al. Structural changes in the renal proximal tubular cells induced by iodinated contrast media. Nephron, v.76, p.96-102, 1997.

THOMSEN, H.S.; HEMMINGSEN, L.; DORPH, $S$. et al. Effects on urine profiles of diatrizoate in hydrated and dehydrated rats: a double crossover study. Acta Radiologica, v.29, p.731-735, 1988.

VIANA, F.A.B. "Fundamentos de Terapêutica Veterinária”. 2000.

http://www.vet.ufmg.br:8080/Portal_Vet/departa mentos/clinica/clinica/documentos/Fundamentos \%20de\%20Terapeutica\%20Veterinaria.pdf/view (15/06/2010).

WALDAY, P.; HEGLUND, I.F.; GOLMAN, K. et al. Renal effects of lodixanol in experimental animals. Acta Radiologica, v.36, p. 204-212, 1995.

WANG, Y-X.J. ; JIA, Y-F ; CHEN, K-M. et al. Radiographic contrast media induced nephropathy: experimental observations and the protective effect of calcium channel blockers. The British Journal of Radiology, v.74, p.1103-1108, 2001. 